\title{
Agent-based modelling of quality management effects on organizational productivity
}

\author{
B Jamshidnezhad ${ }^{1 *}$ and KM Carley ${ }^{2}$ \\ ${ }^{I}$ The University of Melbourne, Parkville, Australia; and ${ }^{2}$ Carnegie Mellon University, Pittsburgh, PA, USA
}

This paper presents the application of agent-based simulation as a modelling metaphor for investigating the relationship between quality management $(\mathrm{QM})$ and organizational productivity. The effects of $\mathrm{QM}$ on organizational productivity are traditionally researched by inductive reasoning through statistical models. Adopting a macro (system) level, top-down approach, statistical models fall short of providing an explanatory account of micro-level factors like individual's problem-solving characteristics or customer requirements complexity, because organizations are considered as black boxes in such models and hence constructs of QM are defined at an organizational level. The question is how an explanatory, bottom-up account of QM effects can be provided. By virtue of the agent-based modelling paradigm, an innovative model, fundamentally different from the dominant statistical models is presented to fill this gap. Regarding individuals' characteristics, results show that a well-balanced organization comprised of similar agents (in terms of agents' problem-solving time and accuracy) outperforms other scenarios. Furthermore, from the results for varying complexity of customer requirements, it can be argued that more intricacy does not always lead to less productivity. Moreover, the usefulness of quality leadership represented as a reinforcement learning algorithm is reduced in comparison to a random algorithm when the complexity of customer requirements increases. The results have been validated by face validation and real data.

Journal of Simulation (2015) 9(1), 73-82. doi:10.1057/jos.2014.26; published online 3 October 2014

Keywords: simulation; organizational studies; management

\section{Introduction}

The interrelationship between quality management $(\mathrm{QM})$ and organizational productivity has long been of interest to academics and practitioners. QM-productivity research is mainly conducted by inductive reasoning based on linear statistical models. Sometimes referred to as Input-Output models, statistical models generally are applied to model the relationship between outputs and inputs of a system, because organizations are considered as black boxes and hence constructs of a theoretical framework like QM are defined at an organizational level. That is why they seem to be inappropriate for explaining or understanding the behaviour of a system by which the underlying mechanism of a system can be explained. In the case of QM, statistical models do not clarify the links between micro-level gradual improvements recommended in quality models (ie, by Plan-Do-Check-Act (PDCA) cycles, or training) with macro-level organizational productivity. In other words, they do not provide the dynamic mechanisms underlying the micro-macro level linkages in QM.

The objective of this research is to develop a computational model by means of agent-based modelling (ABM) to provide an explanatory account for QM effects on productivity. In particular, some frequently neglected aspects of QM like individuals' characteristics and customer requirement complexity are examined. These are micro-level factors that

*Correspondence: B Jamshidnezhad, Department of Mechanical Engineering, The University of Melbourne, Parkville 3052, VIC, Australia.

E-mail: bizhan1847@gmail.com probably cannot be explained by statistical models because of their embedded limitation. To describe the developed model called Multi Agent Quality Model (MAQM), its theoretical background is discussed first, followed by the computational model and then the results.

\section{Background}

Agent-based models have been increasingly used over the past two decades to support organizational analysis. These models support reasoning about micro-level activity for heterogeneous sets of actors (Gilbert, 2007) who may have constraints on their behaviour, at the same time as supporting the consequent macro results that emerge from micro-level preferences (Schelling, 1978). As such, ABM has been used to analyse a wide range of behaviour. From an organizational perspective, applications have included information diffusion (Carley et al, 2009), terrorist groups (Moon and Carley, 2007), communication networks (Schreiber and Carley, 2012) organizations under stress (Lin et al, 2006), and many more.

ABM seems to be an appropriate modelling paradigm for QM. The reasons Macal and North (2007) express for using $\mathrm{ABM}$ are present in $\mathrm{QM}$ research. First, there are employees, teams, and departments in an organization, naturally representing agents at different levels. Second, the bottom-up approach embedded in $\mathrm{ABM}$ is consistent with the practice of $\mathrm{QM}$ in an organization, because management of quality is realized 
through a hierarchy of organizational processes in response to a QM-related event such as a customer complaint. These organizational processes at micro levels are summed as the organizational productivity at the macro level. Third, if QM is construed as a method for enabling organizational change (Varman and Chakrabarti, 2004), improvement could be interpreted as an outcome of multiple interacting people (Dooley, 1995; Dooley, 1997). In Complex Adaptive Systems (CAS) or complexity theory parlance, organizational productivity is an 'emergent' behaviour (Lissack, 1999). To summarize, the practice of QM in organizations does lend itself to ABM.

Despite their pervasiveness and without ignoring their profound impacts, statistical models fall short of providing microlevel explanation for QM effects. This is because they are based on an equation-based, top-down modelling approach but micro level explanation does not lend itself to top-down modelling. That is why micro level factors like the way quality leadership affects organizations, the impacts of information analysis and customer focus are all missing in the current literature of QM research. The possibility of developing a mechanism-based model through ABM (Carley and Prietula, 1994) offers new rooms for researching micro-level constructs of QM.

Information analysis is considered among the important constructs of QM in empirical models (for instance, see Beer, 2003; Singh, 2008). Within an information-processing view of an organization, accurate information processing within each department and sound information transfer between and within departments are crucial for making correct decisions. They also directly affect coordinating and controlling activities which are ultimately reflected in organizational performance (Burton, 1995). In this regard, the role of open horizontal communication and other formalized coordination mechanisms are stressed in QM literature (Flynn, 1994; Powell, 1995). However, the capabilities of employees like problem-solving time and accuracy have not been seen in the QM literature. Rather, the emphasis is put on training and motivation as crucial factors in improving organization productivity.

Another important construct of QM is customer focus which has been repeatedly considered in empirical models. It is defined as the establishment of links between customer needs and satisfaction and internal processes (Sousa, 2003). In the QM literature, it is stressed that identifying customer requirements is an important success factor of quality initiatives and any failure in this activity can lead to solving a wrong question, no matter how well it is done (Stahl, 1999). One critical factor in achieving customer satisfaction is the complexity of customer requirements. However, there is an apparent dearth of researches that address customer requirement complexity. Apart from Benson et al's (1991) study modelling product complexity as a contextual variable, to the knowledge of the authors, customer requirements complexity has not been considered as an important element of QM, probably because of modelling difficulties.

In summary, although there are plenty of research works in the literature of QM adopting a top-down, equation-based approach (ie, statistical models), it seems that there is a lack of bottom-up, explanatory models to address the effects of micro-level factors like individual's characteristics on organizational productivity. This research is an attempt to plug this gap by means of ABM. In the next section, the theoretical foundations of MAQM are explained.

\section{Theoretical foundations of MAQM}

The development of MAQM is based on a fusion of learning concepts from three areas: organization science, QM, and multiagent systems. The existence of analogies in these three areas around the concept of learning makes it possible (and plausible) to build a computational model for QM effects on productivity. Explaining organizational learning theories is not within the scope of this paper and thus is not covered here. However, the important point is that QM can be interpreted within the framework of organization learning (Dooley, 1995; Hackman and Wageman 1995; Lundberg, 1995; Dooley, 1997; Beer, 2003). Organizational learning is a process of having an experience, making new observations and reflecting on that experience, forming generalizations from experiences and testing them in new situations (Levitt and March, 1996; Dodgson, 1993); it can be in the form of people's knowledge acquisition (Simon, 1991) or standardized routines (Argyris, 1999). Among the recommended tools for QM implementation, PDCA is an outstanding example of this kind of learning. Learning in QM happens within each person, department, and working process (Hackman and Wageman, 1995). It includes learning from past experiences of success or failure, adaptation to and satisfying customer wants, learning from customer complaints, and learning from continuous improvement of internal processes or necessary corrective actions within an organization. This conception of QM as a learning mechanism acts as the bedrock of MAQM which is explained in the next section.

\section{Computational model of MAQM}

Contemplating its prescriptions about how to manage an organization from a quality standpoint, QM can be modelled as a set of behaviours (Edwards et al, 1998). This is what MAQM is founded upon in which an organization is conceived as a goaldirected, routine-guided, experiential learning system. In Figure 1, the structure of MAQM is shown.

The organization, hereinafter called organization, is comprised of five intelligent agents, interacting through two business pro-

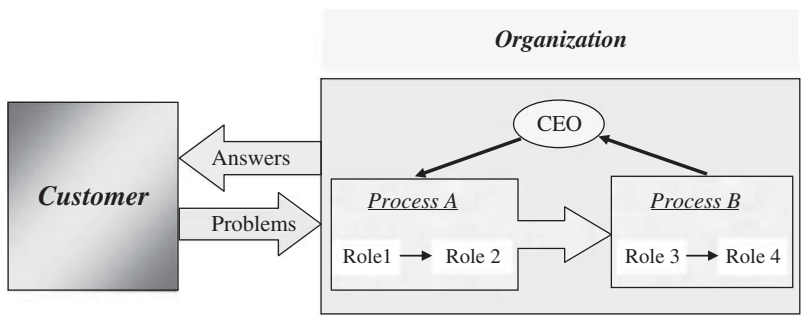

Figure 1 Multi agent quality management model (MAQM). 
cesses. The director of organization, hereinafter called $C E O$, is responsible for improving organization. The environment consists of a customer agent, called customer hereinafter, challenging the organization by generating random problems. These problems model the changing nature of customer requirements or corrective actions or improvements needed for business processes. They are comprised of seven binary variables $X_{0}, X_{1}, X_{2}$, $X_{3}, X_{4}, X_{5}, X_{6}$; each can take one or two as their value with equal probability. The size of problems can be selected more than seven but it would increase simulation time. Tasks are classification choice tasks in which the solution is one or two depending on the applied decision rule. This type of symbolically modelling customer wants, called canonical tasks (Moss, 2000), is a natural method of representing human knowledge and intelligence (Newell and Simon, 1976; Newell, 1990). The solution is a function of these variables henceforth called decision rule. In pursuing customer satisfaction, organization should promptly respond to these problems by finding the solution. This calls for solving problems with highest effectiveness and efficiency. The accuracy of answers is a measure of effectiveness, while total processing time represents the efficiency of organization.

Through solving different problems, $C E O$ and the analyst agents (one located at each role) improve their performance by learning from the memory implemented as lookup tables. $C E O$ has another learning algorithm based on reinforcement learning (RL) in order to improve organization and perform the quality leadership duty, because within the quality management framework one of the management responsibilities is to review the quality management system periodically and to align the organization towards customer requirements. Therefore, customer feedback plays a key role in deciding on required actions for improvement. This is the role the RL algorithm is playing in MAQM with the additional advantage of simultaneously optimizing efficiency (ie, minimizing the total time). To fulfil this responsibility, $C E O$ tries to improve organization by modifying the business processes and changing the agents' roles within and between processes. In reality, organizations have internal measurement systems to evaluate their own business processes and in the case of nonconformity or the need for improvement, an action is necessary. The dynamics of the simulation is as follows:

(1) Customer generates a random problem and sends it to organization.

(2) $C E O$ collects a problem and sends it to process A. The agent at role one starts solving the problem.

(3) Each agent receives the problem and depending on its role looks at certain bits to provide the solution from its memory and sends the solution to its successor. The duration of problem solving depends on the number of bits each agent encounters as well as the agents' characteristics.

(4) $C E O$ provides the overall answer to the problem and passes it to customer.
(5) $C E O$ updates itself and the other agents according to the feedback of customer; all agents update their memory based on the feedback.

(6) According to a fixed frequency, $C E O$ tries to make some changes to organization (based on the RL algorithm) to improve the performance.

To measure organization efficiency, associated with each problem are time fields, including problem generation time and problem final time. The problem generation time is set at first and then is accumulated after processing by agents or passing through the network at each role. The transfer time of problems between agents is fixed at two simulation time units, called ticks. The difference between the generation time and the final time is a measure of efficiency. By calculating the ratio of the number of correct answers to total problem-solving time, organization measures productivity. This value is multiplied by 100 for normalization purposes. The performance measure defined here seems to be an appropriate measure because 'the ratio of results achieved to resources consumed, is an appropriate and fundamental criterion for all of organizational decisions' (Simon, 1997, p 277). Not every agent has access to all bits of a problem due to division of work in an organization. The assignment of problem bits is shown at Table 1. This allocation scheme, representing the process approach in QM, is completely arbitrary. Note, MAQM is an intellective model, and as such is intended to capture the key features of the system and not to provide exact descriptive realism. As our goal is to ask a simple 'what-if' question, and not to assess the specific processes in a specific organization, such a high level and abstract model seems warranted.

The $C E O$ tries to improve organization by modifying processes A and B in every 500 ticks, where it changes the agent roles within and between these processes. The list of its actions is presented in Table 2. For instance, assume the layout of a simulation run is DBCA, meaning agent $\mathrm{D}$ at role one; agent $\mathrm{B}$ at role two; agent $\mathrm{C}$ at role three; and agent $\mathrm{A}$ at role four.

Table 1 Problem decomposition structure

\begin{tabular}{ll}
\hline Role 1 & $x_{0}, x_{1}, x_{2}$ \\
Role 2 & $x_{5}$, output of role 1 \\
Role 3 & $x_{3}, x_{4}$, output of role 2 \\
Role 4 & $x_{6}$, output of role 3 \\
CEO & output of role 2, output of role 4 \\
\hline
\end{tabular}

Table 2 CEO actions used for RL

\begin{tabular}{ll}
\hline Action & Description \\
\hline 1 & Swap agents at roles 1 and 2 \\
2 & Swap agents at roles 3 and 4 \\
3 & Swap agents at roles 2 and 3 \\
4 & Swap agents at roles 1 and 4 \\
5 & Swap agents at roles 2 and 4 \\
6 & Swap agents at roles 1 and 3 \\
7 & Do nothing \\
\hline
\end{tabular}


By taking action 3 for example, $C E O$ changes the layout of organization to DCBA. The consequence of such an action (the reward) appears in the form of an increase or decrease of average performance, incremented by the following equation:

$$
\overline{r_{t+1}}=\overline{r_{t}}+\alpha\left[r_{t}-\overline{r_{t}}\right]
$$

where $\alpha$ is step-size parameter; $r_{t}$ is the productivity level of the current run; and $\overline{r_{t}}$ is the average productivity level until time $t$. Each action has a numeric preference level $\left(p_{t}(a)\right)$, incremented in each decision making stage according to the following equation:

$$
p_{t+1}(a)=p_{t}(a)+\beta\left[r_{t}-\overline{r_{t}}\right]
$$

where $\beta$ is another step-size parameter. The preference of selecting successful actions rises gradually, which in turn increases the probability of their own selection according to the Gibbs distribution:

$$
\operatorname{Pr}\left\{a_{t}=a\right\}=\frac{e^{p_{t}(a)}}{\sum_{b=1}^{n} e^{p_{t}(b)}}
$$

Equation (1) indicates the way $C E O$ senses customer satisfaction over time, while Equations (2) and (3) represent how these sensory data are translated into a cognitive schema for decision making.

The default parameters of the simulation are shown in Table 3. These show agent A processes data quickest but has a poor 'memory' for the consequences of past decisions, in contrast to agent $\mathrm{D}$ who is slow at decision making but has a perfect memory.

Cut-off values determine the correct answers of each problem. They are set so that the probability of being 'one' or 'two' is equal to 0.5 . For example, with the linear decision rule for there to be a majority, the number of ones should be at least four or equivalently the number of twos should be no more than three. The probability distribution of the linear decision rule is:

$$
\operatorname{Pr}\left(Y_{\text {Linear }}=a+7\right)=\left(\begin{array}{l}
7 \\
a
\end{array}\right)\left(\frac{1}{2}\right)^{7}
$$

Table 3 Default parameters of simulation runs

\begin{tabular}{ll}
\hline $\begin{array}{l}\text { Problem-solving time (simulation } \\
\text { ticks/bit) }\end{array}$ & $\begin{array}{l}\mathrm{A}=1, \mathrm{~B}=2, \mathrm{C}=3, \mathrm{D}=4, \\
C E O=1\end{array}$ \\
$\begin{array}{l}\text { Ratio of memory forgetfulness } \\
\text { (between 0 and 1) }\end{array}$ & $\mathrm{A}=0.3, \mathrm{~B}=0.2, \mathrm{C}=0.1$, \\
$\begin{array}{l}\text { Transfer time of problems between } \\
\text { agents (simulation ticks) }\end{array}$ & 2 (constant for all activities) \\
$\begin{array}{l}\text { Simulation run time (simulation ticks) } \\
\text { Action preference step-size }\end{array}$ & 8000000 \\
parameter $(\beta)$ & 0.2 \\
$\begin{array}{l}\text { Reward step-size parameter }(\alpha) \\
\text { Linear decision rule }\end{array}$ & 0.1 \\
& $\begin{array}{l}Y=\Sigma_{i=0}^{6} X_{i} \text { with cut-off } \\
\text { value }=10\end{array}$ \\
\hline
\end{tabular}

Where $a$ is the number of twos. Therefore, the cut-off value can be calculated as follows:

$$
\begin{aligned}
\operatorname{Pr}\{a \leqslant 3\} & =\operatorname{Pr}\left\{Y_{\text {Linear }} \leqslant c u t-\text { off }\right\} \\
& =0.5 \rightarrow \text { cut }- \text { off }_{\text {Linear }}=10
\end{aligned}
$$

The cut-off value for any other decision rule is calculated in the same way depending on their probability function. Total time of problem-solving can be calculated by taking into account agents' processing time (including $C E O$ ) and the number of bits they process (according to Table 1). As shown in Table 4 for a sample problem, the total time is accumulated as organization solves more problems in a simulation period.

The structure of the programme is presented in Table 5. This structure was implemented in REPAST in which some JAVA snippets have been added. Agents are updated every 500 ticks.

\section{Virtual experiment}

The characteristics of the experimental designs for each scenario are shown in Table 6. Customer requirement complexity has been modelled as the complexity of the decision rules. For the individual's characteristics, three different combinations of agents' processing time and forgetfulness are tested.

\section{Results and discussions}

In this section, hypothetical scenarios will be examined using MAQM so as to provide insights into the QM-productivity relationship. MAQM acts as a virtual laboratory to collect data about organizational activity given the scenarios. The simulation programme has been verified manually by checking the performance of the programme with different parameters. One-way analysis of variance is applied to analyse the results, where it is assumed that there is a linear relationship between independent and dependent variables.

\subsection{Effects of individuals' characteristics}

To evaluate the effects of agent characteristics in MAQM, a set of experiments is conducted in which individual's characteristics,

Table 4 An example of problem solving

\begin{tabular}{lccc}
\hline Role & $\begin{array}{c}\text { Sub- } \\
\text { problem }\end{array}$ & $\begin{array}{c}\text { Processing time (number } \\
\text { of bits times the agent's } \\
\text { procession time }\end{array}$ & Total time \\
\hline $1 \sim \mathrm{B}$ & {$[1,2,1]$} & $2 \times 3=6$ & 6 \\
$2 \sim \mathrm{C}$ & {$[1,2]^{*}$} & $3 \times 2=6$ & $6+6+2=14$ \\
$3 \sim \mathrm{A}$ & {$[2,2,1]$} & $1 \times 3=3$ & $14+3+2=19$ \\
$4 \sim \mathrm{D}$ & {$[2,1]$} & $4 \times 2=8$ & $19+8+2=29$ \\
CEO & {$[1,1]$} & $1 \times 2=2$ & $29+2+2=33$ \\
\hline
\end{tabular}

Note: The problem is $[1,2,1,1,2,2,2]$.

*Italic numbers are processed information from previous roles. 
Table 5 Class definitions in the programme

\begin{tabular}{|c|c|c|c|}
\hline Class Name & Super class Name & Variables & Methods \\
\hline SimpleAgent & - & $\begin{array}{l}\text { agentLoc, absoluteMemory, buffer, } \\
\text { currentMemory, isBusy, oldMemory, } \\
\text { solvedProblems, trainingMemory }\end{array}$ & $\begin{array}{l}\text { genericUpdate(), genericUpdateForTraining(), } \\
\text { solve(byte), step(), train(byte), transfer } \\
\text { (Problem), LinkedList), update(), } \\
\text { updateAbsoluteMemory() }\end{array}$ \\
\hline$A, B, C, D$ & SimpleAgent & negligence, problem_Solving_Time & step(), update() \\
\hline$C E O$ & SimpleAgent & $\begin{array}{l}\text { correctAnswers, correctTime, count, currentAction, } \\
\text { currentState, negligence, oldAction, oldState, } \\
\text { preferenceValue, problem_Solving_Time, } \\
\text { step_Size_preference, step_Size_Reward, } \\
\text { totalTime, uchangedBits, agentList, } \\
\text { averageReward, mapOfActions, preferenceMatrix, } \\
\text { probabilityMatrix }\end{array}$ & $\begin{array}{l}\text { act (), collect(), findRow(short), improve(), } \\
\text { step }(), \text { update() }\end{array}$ \\
\hline Customer & SimpleAgent & problemsGenerated & generateProblem(), update() \\
\hline Problem & - & problem, time & $\operatorname{ini}()$ \\
\hline ModelInitializer & - & agentList, agentLoc & initializeModel() \\
\hline
\end{tabular}

Table 6 The experimental designs of the scenarios

\begin{tabular}{|c|c|c|c|c|}
\hline Scenario & $\begin{array}{l}\text { Dependent } \\
\text { variable }\end{array}$ & $\begin{array}{l}\text { Independent } \\
\text { variables }\end{array}$ & $\begin{array}{l}\text { Number } \\
\text { of levels }\end{array}$ & $\begin{array}{l}\text { Runs } \\
\text { per cell }\end{array}$ \\
\hline $\begin{array}{l}\text { Individuals' } \\
\text { characteristics }\end{array}$ & productivity & Agent type & 3 & 10 \\
\hline $\begin{array}{l}\text { Customer } \\
\text { requirement } \\
\text { complexity }\end{array}$ & productivity & $\begin{array}{l}\text { Decision } \\
\text { rule }\end{array}$ & 4 & 10 \\
\hline
\end{tabular}

called 'agent type' in the experimental setting, are varied. This factor will be evaluated for several decision rules to determine if there are any confounding effects among agent types and customer requirement complexity.

Agent type represents hypothetical scenarios about agents' characteristics in terms of information processing time and forgetfulness (as a measure of accuracy). The first level is the default scenario as outlined in Table 3 in which there exists one quick and inaccurate agent $\mathrm{A}$ and one slow and accurate agent $\mathrm{D}$ and the other agents, B and C, are in between these extremes. The second level characterizes the scenario in which there is an excellent agent, A, who is both quick and accurate along with a slow and forgetful agent, D. It is called excellent-forgetful scenario. The final scenario, average scenario, models the hypothetical situation where agents are all the same and show average performance both in time and forgetfulness. In this scenario, all problem-solving times are set to two ticks and the forgetfulness factors are set to 0.15 which is the average of forgetfulness rates of all agents.

The results of the single-factor analysis of variance, presented in Table 7, reveal that there is no evidence to accept the null hypothesis of similar effects of agent types, because significance level is less than selected type I error level (ie, $\alpha=5 \%$ ). Therefore, the alternative hypothesis is accepted. The pair-wise comparison tests suggest that average agents outperform other scenarios as can be seen in Table 8 and Figure 2. That the average agent scenario is the best arrangement of agents implies that the
Table 7 Tests of between-subjects effects for agent type scenario

\begin{tabular}{lrrrrr}
\hline Source & $\begin{array}{c}\text { Type III sum } \\
\text { of squares }\end{array}$ & df & \multicolumn{1}{c}{$\begin{array}{c}\text { Mean } \\
\text { square }\end{array}$} & $F$ & Significance $^{\dagger}$ \\
\hline $\begin{array}{l}\text { Corrected } \\
\text { Model }\end{array}$ & $0.215^{*}$ & 2 & 0.107 & 20.474 & 0.000 \\
Intercept & 278.701 & 1 & 278.701 & 53187.014 & 0.000 \\
Agent & 0.215 & 2 & 0.107 & 20.474 & 0.000 \\
type & & & & & \\
Error & 0.692 & 132 & 0.005 & & \\
Total & 279.607 & 135 & & & \\
Corrected & 0.906 & 134 & & & \\
total & & & & & \\
\hline
\end{tabular}

$* R^{2} 0.237$ (Adjusted $R^{2}=0.225$ ).

${ }^{\dagger}$ Computed using $\alpha=0.05$.

performance level in MAQM is dependent on the smoothness of flow of problems within the processes like a production line, even with the performance measure being a function of accuracy and efficiency. In other words, eliminating any 'bottleneck', be it in the processing time or accuracy of agents, plays a key role in performance improvement. This suggests an insight into organization policies related to staffing, training and organizing; that is, a well-balanced set of working teams boosts performance.

The mean productivity of default agents is statistically less than that of the excellent-forgetful scenario. Within the framework of MAQM where agents do not have any cooperation and coordination, agents cannot take advantage of each other's strength and that is why the average productivity for default agents where there is no excellent agent in terms of both forgetfulness and problem-solving time is the worst scenario. This can be explained from another viewpoint. The $C E O$ tries to make some changes in the configuration and learns from them. In fact, any change in the productivity is sensed and then converted into some changes in the overall average productivity and the usefulness of each action in each state (ie, preference levels). The greater the changes in the productivity, the bigger their 
Table 8 Pair-wise comparison for the agent type scenario

\begin{tabular}{llcc}
\hline (I) Agent type & $(J)$ Agent type & Mean difference $(I-J)$ & Standard error \\
\hline Default & Excellent-stupid & $-0.03606724^{*}$ & 0.013091052 \\
& Average agents & $-0.09662524^{*}$ & 0.017196380 \\
Excellent-stupid & Default & $0.03606724^{*}$ & 0.013091052 \\
& Average agents & $-0.06055800^{*}$ & 0.015217663 \\
Average agents & Default & $0.09662524^{*}$ & 0.017196380 \\
& Excellent-stupid & $0.06055800^{*}$ & 0.015217663 \\
\hline
\end{tabular}

Source: Based on observed means.

Note: The error term is Mean Square(Error) $=0.005$.

*The mean difference is significant at the 0.05 level.

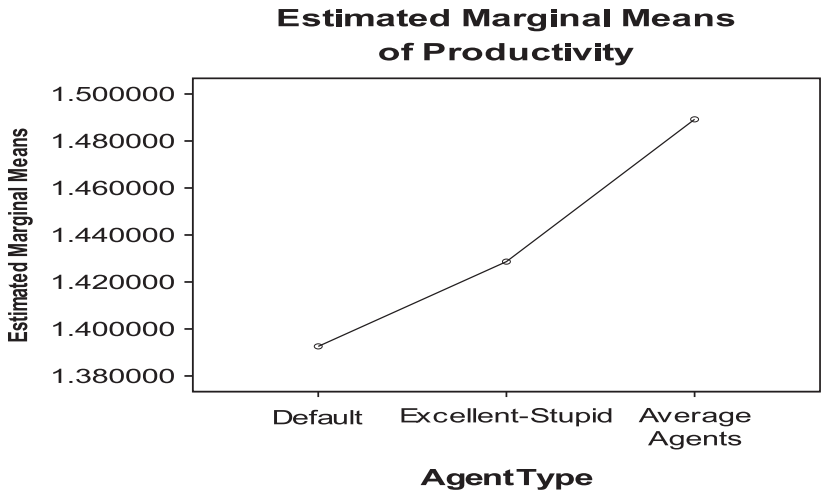

Figure 2 Performance of agent types.

effects on the CEO's cognitive map. It is expected that in the excellent-forgetful scenario, where agent $A$ is quick and accurate and agent $\mathrm{D}$ is slow and inaccurate, more extreme changes in the productivity are seen when changing agents between roles and consequently the $C E O$ can detect the best configuration quickly. In contrast, the changes in the productivity are less perceptible in the default scenario and thus the $C E O$ requires more time to converge towards the best actions.

In the average agent scenario, the $C E O$ needs fewer tests to learn the best configuration than in the other two scenarios, because there is only one arrangement of agents (ie, state). From the $C E O$ 's viewpoint, its cognitive map takes less time to converge to a special arrangement of agents in the average scenario where the exploitation is complete. In the average scenario, the CEO need not engage in exploration, as there is only one state.

\subsection{Effects of customer requirements complexity}

The architecture of MAQM offers the possibility of modelling the complexity of customer requirements through the complexity of decision rules. The complexity within MAQM originates from the problem bits sample space that could be binary, ternary or some other form, their associated probabilities, and decision rules' functions. In MAQM, the problem space is binary and fixed in which each bit is a Bernoulli random variable with the parameter of 0.5 . Consequently, to examine the effects of customer requirement complexity, organization is exposed to
Table 9 Characteristics of decision rules for binary tasks

\begin{tabular}{lrr}
\hline Solution & Entropy & $\begin{array}{r}\text { Cut- } \\
\text { off }\end{array}$ \\
\hline Linear: $Y=\Sigma_{i=0}^{6} X_{i}$ & 2.4466 & 10 \\
Third Degree: $Y=X_{0}+X_{1} X_{2} X_{3}+X_{4} X_{5} X_{6}$ & 3.4135 & 7 \\
Mixed Third Degree: $Y=X_{0}+X_{1}+X_{2} X_{3}+X_{4} X_{5} X_{6}$ & 3.2171 & 8 \\
Weighted Linear: & 3.8038 & 25 \\
$Y=X_{0}+2 X_{1}+2 X_{2}+2 X_{3}+4 X_{4}+3 X_{5}+2 X_{6}$ & \\
\hline
\end{tabular}

more complexity by applying new decision rules. The specifications of these rules including their associated information entropy and cut-off values are summarized in Table 9. Since information entropy is a measure of complexity of search space and a function of range of possibilities and their associated probabilities (Ross, 2002) and organiztion's task is to discover decision rules, it has been selected as measure of complexity of decision rules. It is calculated by:

$$
H(X)=-\sum_{i=1}^{n} p_{i} \log p_{i}
$$

where $p_{i}$ is the probability of taking value $i$ for random variable $X$ (Shannon, 2001).

As seen in Figure 3, organization's productivity is better with the linear decision rule in comparison to the others. Furthermore, weighted linear and third degree rules, despite having higher entropy, outperform the mixed third degree decision rule. This is contrary to the expectation that more complexity leads to less performance. Consequently, it seems that drawing a general rule out of these results based on the entropy values is difficult.

The knowledge level gained by MAQM is bounded by agents' capabilities and the RL algorithm and consequently MAQM has limited capabilities in targeting correct answers. In fact, since learning in MAQM is incomplete, there is always a degree of error when $C E O$ provides answers to customer problems and as a result $C E O$ lacks prediction accuracy. However, the relative performance of MAQM under various decision rules depicted in Figure 3 reveals that complexity is not the only important parameter affecting performance and there may be some more confounding parameters related to decision rules such as their 


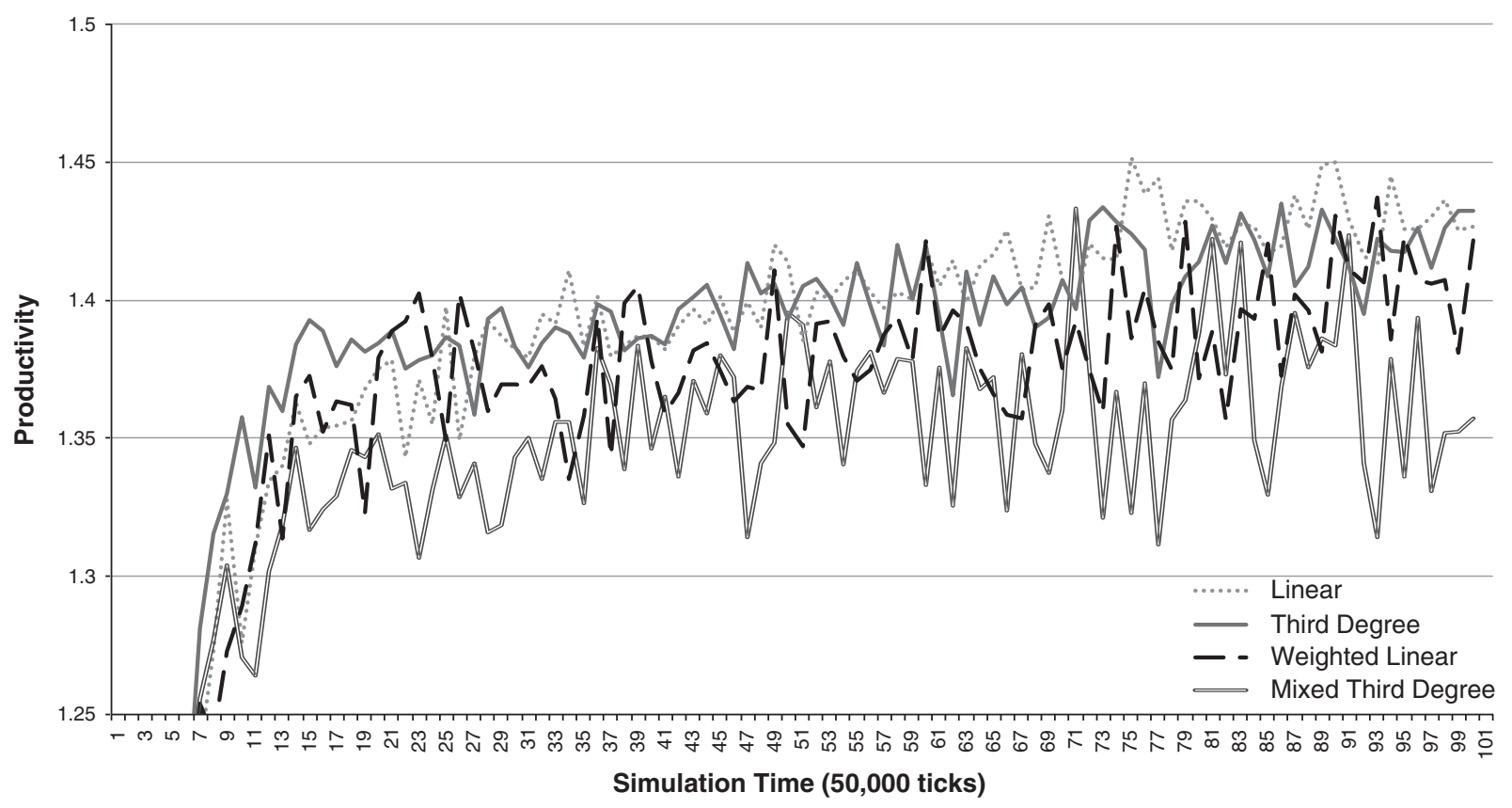

Figure 3 Comparison of productivity level for RL with different decision rules.

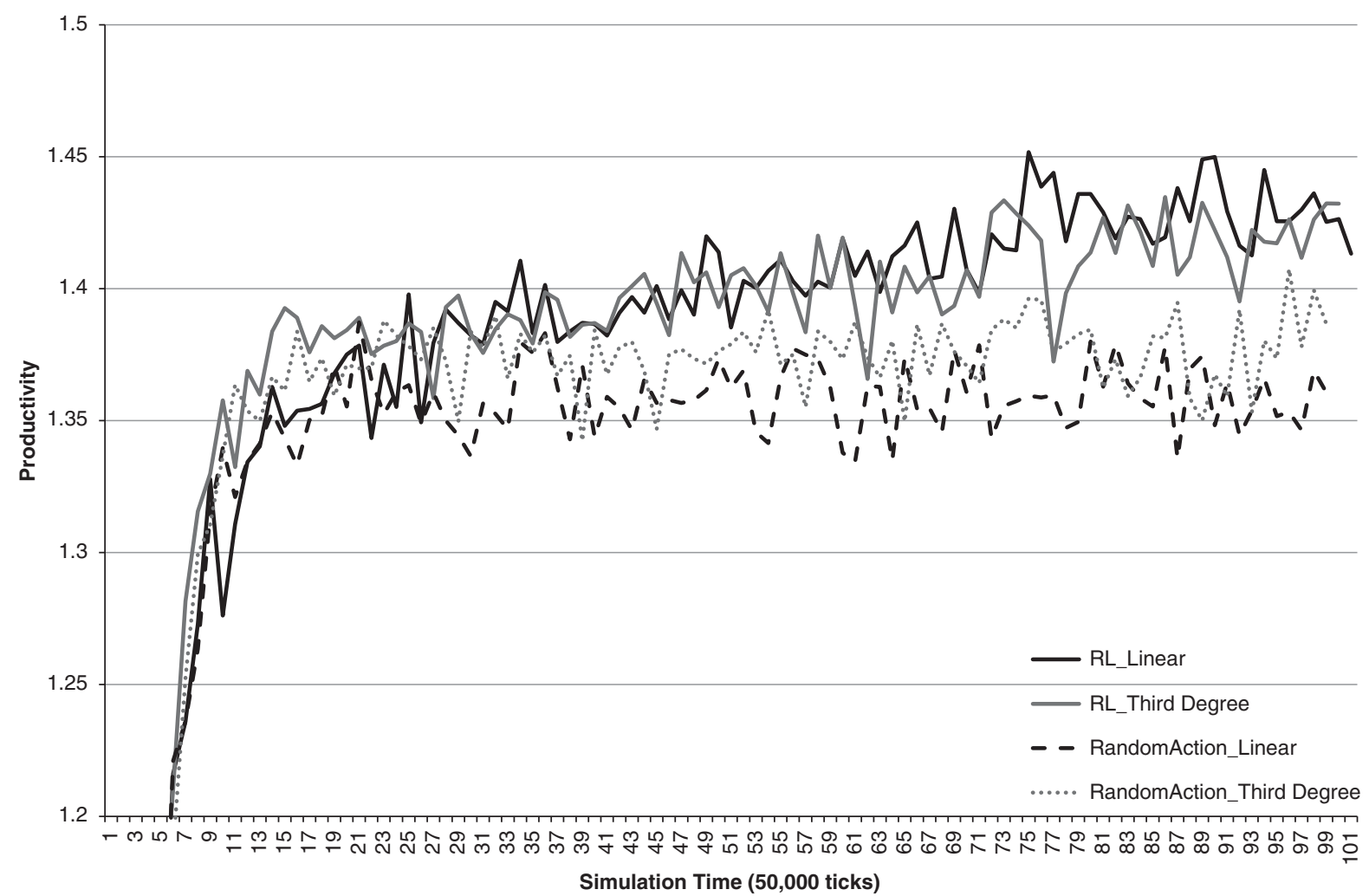

Figure 4 Comparison of RL and Random-Action for the third degree and linear decision rules.

functional form or the problem decomposition scheme (the way problem bits are distributed among various roles) that can be investigated in future research projects.
The incompleteness of learning is more critical when decision rules are more complex, resulting in loss of performance. Figure 4 depicts an example where the linear and the third degree 


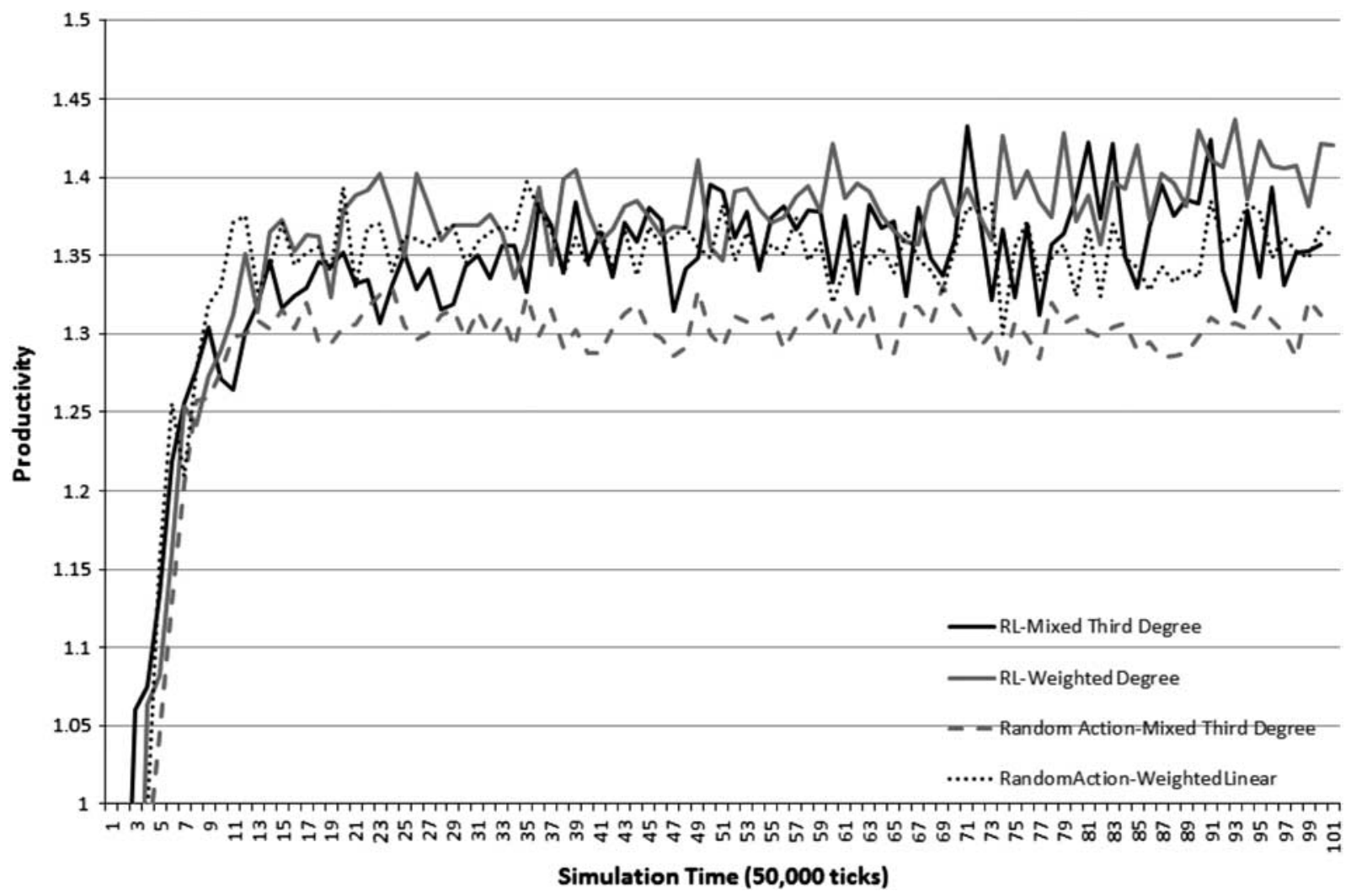

Figure 5 Comparison of RL and Random-Action for the mixed third degree and weighted linear decision rules.

decision rules are compared. The difference between RL and Random-Action algorithms by which $C E O$ randomly selects an action is less with the third degree decision rule in comparison to that of the linear decision rule. Thus, with more complex problems, the difference between RL and Random-Action algorithms decreases. As another example, the case of the semi-linear and the mixed third-degree decision rules is presented in Figure 5, reflecting a similar trend.

From the results presented in this section, it can be inferred that CEO's quality leadership represented by RL algorithm can be more fruitful than Random-Action when customer requirements are less complex. As regards the RL algorithm, although in most cases complexity is detrimental to performance, there may be some confounding effects by the form of decision rules or the problem decomposition structure. These aspects need more investigation.

\section{Validation and verification of the results}

Substantiating that a model as a representation of a system is accurate requires validation and verification (Balci, 1994). Validation contrasts with verification in that verification reveals how appropriately the assumptions and model constructs are translated into the computational model while validation deals with how outputs of a model correspond to the target system (Casti, 1997).
In other words, verification is an indicator of internal consistency while validation is a yardstick of external consistency. As regards MAQM, the behaviour of MAQM has been verified by several tests such as consistency checking and data flow analysis (see Balci, 1994 for more details); therefore, the issue of validation comes to the fore.

Different questions of validity have been raised against agentbased models depending on the objective of modelling and the subject of simulation (Burton and Obel, 1995; Casti, 1997). Building models, in general, can be aimed at describing a phenomenon (explanatory) or predicting the future behaviour of a system (predictive) (Casti, 1997). Whether validation is more related to descriptiveness or predictive power is arguable (Moss and Davidsson, 2001).

Depending on the objective of modelling, the validation method differs. In explanatory models, the exact prediction of the target system is not of interest either because of the nature of explanatory research questions or difficulty in considering the details of the target system (Bankes, 1993). Rather, the emphasis is put on discovering the underlying mechanism of a phenomenon and how the macro-level outcomes can be explained by micro-level mechanisms.

The subject of simulation has its own implications as well. Since a simulation model should be validated against its referent (Balci, 1994; Casti, 1997), when the phenomenon under study is real and concrete, consistency with the reality is the main 
Table 10 Students grades summary for different scenarios

\begin{tabular}{|c|c|c|c|c|}
\hline \multirow[b]{2}{*}{$\begin{array}{l}\text { Project } \\
\text { Complexity }\end{array}$} & \multicolumn{3}{|c|}{ Team composition } & \multirow{2}{*}{$\begin{array}{l}\text { Average } \\
\text { grade }\end{array}$} \\
\hline & Default & Average & $\begin{array}{l}\text { Excellent- } \\
\text { stupid }\end{array}$ & \\
\hline Low & NA & NA & 88,78 & 83 \\
\hline Medium & $90,69,58,79,65,62$ & 54 & NA & 68.1 \\
\hline High & NA & $82,70,87,75$ & $65,65,70,65$ & 72.4 \\
\hline Average & 70.5 & 73.6 & 71.8 & \\
\hline Grade & & & & \\
\hline
\end{tabular}

criterion for model validation. However, when the subject of simulation is abstract (ie, a model), defining any quantitative measure seems to be impossible. Instead, this requires validation based on expert judgment, which is called face validation (Balci, 1994; Zacharias et al, 2008).

For validating MAQM, the above-mentioned issues should be addressed, namely the objective of simulation and its referent system. The objective of developing MAQM is to investigate the effects of QM on organizations. From this viewpoint, MAQM is an explanatory model, because it is not intended to predict future behaviours of its referent system. Therefore, to validate MAQM as a good explanatory model, perfect match between its characteristics and the referent system is not necessary; rather 'a certain level of accuracy is needed in order to take the simulation seriously' (Casti, 1997, p 75) which is mostly conducted by face validation. Face validation of MAQM by means of expert judgment reveals that it represents simply the practice of QM in organizations; therefore it is a 'good' explanatory model for its purposes.

Validation of MAQM results involves assessing their credibility against the subject of simulation. The target system of MAQM is not a real organization but rather an abstraction of reality of an organization from the viewpoint of QM. The outputs of MAQM are results about hypothetical scenarios abstractly imitating QM implementation in an organization. Therefore, the validity of the results is checked with a real organization of people, where a group of problem solvers are cooperating to solve a problem.

To validate the results, the case of teamwork activity for two postgraduate subjects has been considered. In these subjects, research projects are assigned to groups of 4 students. The task is to conduct these projects based on some assignment briefs. Basically, the structure is the same as MAQM's: a team of four agents trying to solve a problem. The complexity of projects is defined in three levels: low, medium, and high. Table 10 shows the grades each team received after final assessment. Owing to low sample size and lack of information in some scenarios (eg, low project complexity with Default team composition), it is not possible to conduct a thorough analysis of variance. However, the comparison among team composition scenarios reveals that 'Average' teams are performing better than others, followed by Excellent-Stupid teams. These validate the MAQM simulation results and suggest that there may be some psychological grounds for such consistency in the results that need more empirical data to be examined.

Regarding project complexities that mimic the complexity of problems in MAQM, results are not convincingly consistent, as it was the case in MAQM simulation results. On average, students attain better grades with less complex projects but surprisingly teams with very complex projects outperform medium level projects.

\section{Future research}

Reviewing the results of different decision rules reveals that the entropy of decision rules is not the only factor affecting productivity and the functional form of the decision rules may play a role. To explore this factor further, more tests with some advanced analysis of variance techniques, for example, random effects analysis of variance models, are needed in which MAQM is exposed to some randomly-selected decision rules with various functional forms.

Extension of MAQM fundamentals for a network of organizations (a supply chain) is another area for research. Within the current version, there is only one organization, but to represent interactions between a number of organizations, another model could be developed for a supply chain. However, it is noteworthy that the interaction rules among agents may be quite different to those used in this paper and may be based on a market mechanism. This may take the model away from considering quality management principles in isolation.

\section{Conclusions}

In this paper, an agent-based model has been developed to investigate the effects of individuals' characteristics, and customer requirement complexity as the micro-level factors of QM. To examine the effects of these factors, first, the theoretical foundations of MAQM is explained. It is argued that learning is the running thread linking QM with organization science and an ABM paradigm. Second, the structure of MAQM as an intellective, process model of QM is presented. The results of testing the individuals' characteristics scenarios suggest that well-balanced business processes (in terms of problem-solving time and forgetfulness) result in better performance than do other processes. Eliminating bottlenecks in both problem solving time and agent accuracy can improve the balance and so improve performance. We further find that as the complexity of customer requirements increases, performance does not automatically decrease. Rather, the performance impact depends on customer requirement complexity. The simulation results of this research have been validated with real data. The bottom-up approach embedded in MAQM seems to be a credible alternative to the dominant top-down statistical modelling, applied in QM. 


\section{References}

Argyris C (1999). On Organizational Learning, 2nd edn. Blackwell Business: Malden, MA

Balci O (1994). Validation, verification, and testing techniques throughout the life cycle of a simulation study. Annals of Operations Research 53(1-4): 121-173.

Bankes S (1993). Exploratory modeling for policy analysis. Operations Research 41(3): 435-449.

Beer M (2003). Why total quality management programs do not persist: The role of management quality and implications for leading a TQM transformation. Decision Sciences 34(4): 623-642.

Benson PG, Saraph JV and Schroeder RG (1991). The effects of organizational context on quality management: An empirical investigation. Management Science 37(9): 1107-1124.

Burton RM (1995). Strategic Organizational Diagnosis and Design: Developing Theory for Application. Kluwer Academic Publishers: Boston.

Burton RM and Obel B (1995). The validity of computational models in organization science: From model realism to purpose of the model. Computational \& Mathematical Organization Theory 1(1): 57-71.

Carley KM and Prietula MJ (eds) (1994). Computational Organization Theory. N J L Erlbaum Associates: Hillsdale.

Carley KM, Martin MK and Hirshman B (2009). The etiology of social change. Topics in Cognitive Science 1(4): 621-650.

Casti JL (1997). Would-be Worlds: How Simulation is Changing the Frontiers of Science. John Wiley: New York.

Dodgson M (1993). Organizational learning: A review of some literatures. Organization Studies 14(3): 375-394.

Dooley KJ (1995). TQM, chaos and complexity. Human systems management 14(4): 287-302.

Dooley KJ (1997). A colex adaptive systems model of organization change. Nonlinear Dynamics, Psychology, and Life Sciences 1(1): 69-97.

Edwards P, Collinson M and Rees C (1998). The determinants of employee responses to total quality management: Six case studies. Organization Studies 19(3): 449-475.

Flynn BB (1994). A framework for quality management research and an associated measurement instrument. Journal of Operations Manage ment 11(4): 339.

Gilbert N (2007). Agent-Based Models. Sage Publishers: Thousand Oaks, CA.

Hackman JR and Wageman R (1995). Total quality management: Empirical, conceptual, and practical issues. Administrative Science Quarterly 40(2): 309-342.

Levitt B and March JG (1996). Organizational learning. In: Cohen MD and Sproull LS (eds). Organizational Learning. Sage Publications: Thousand Oaks.

Lin Z, Zhao X, Ismail K and Carley KM (2006). Organizational design and restructuring in response to crises: Lessons from computational modeling and real world cases. Organizational Science 17(5): 598-618.

Lissack MR (1999). Complexity: The science, its vocabulary and its relation to organizations. Emergence 1(1): 110.

Lundberg CC (1995). Learning in and by organizations: Three conceptual issues. International Journal of Organizational Analysis (1993-2002) 3(1): 10-17.
Macal CM and North MJ (2007). Agent-based modeling and simulation: Desktop ABMS. Paper presented at the Simulation Conference, Winter Simulation Conference: Washington DC.

Moss S (2000). Canonical tasks, environments and models for social simulation. Computational and Mathematical Organization Theory 6(3): $249-275$.

Moss S and Davidsson P (2001). Multi-agent-based simulation; second international workshop, MABS 2000, Boston, MA, USA, July: Revised papers Paper presented at the MABS 2000, Berlin, New York.

Moon I and Carley KM (2007). Modeling and simulation of terrorist networks in social and geospatial dimensions. IEEE Intelligent Systems: Special Issue on Special Issue on Social Computing-Sep/ Oct'07 22(5): 40-49.

Newell A (1990). Unified Theories of Cognition. Harvard University Press: Cambridge, MA.

Newell A and Simon HA (1976). Computer science as empirical inquiry: Symbols and search. Communications of the ACM 19(3): 126.

Powell TC (1995). Total quality management as competitive advantage: A review and empirical study. Strategic Management Journal 16(1): $15-37$

Ross SM (2002). A First Course in Probability, 6th edn. Prentice Hall: Upper Saddle River, NJ.

Schelling TC (1978). Micromotives and Macrobehavior, 1st edn. Norton: New York.

Schreiber C and Carley KM (2012). Validating Agent Interactions in Construct Against Empirical Communication Networks Using the Calibrated Grounding Technique. IEEE Transactions on Systems Man and Cybernetics-Part A, 42(6), Institute of Electrical and Electronics Engineers.

Shannon CE (2001). A mathematical theory of communication. SIGMOBILE Mobile Computing and Communication Review 5(1): 3-55.

Simon HA (1991). Bounded rationality and organizational learning. Organization Science 2(1): 125-134.

Simon HA (1997). Administrative Behavior: A Study of DecisionMaking Processes in Administrative Organizations, 4th edn. Free Press: New York.

Singh PJ (2008). Empirical assessment of ISO 9000 related management practices and performance relationships. International Journal of Production Economics 113(1): 40-59.

Sousa R (2003). Linking quality management to manufacturing strategy: An empirical investigation of customer focus practices. Journal of Operations Management 21(1): 1-18.

Stahl MJ (1999). Perspectives in Total Quality. Blackwell in association with ASQ Quality Press: Malden, MA, Oxford.

Varman R and Chakrabarti M (2004). Contradictions of democracy in a workers' cooperative. Organization Studies 25(2): 183-208.

Zacharias G, MacMillan J and Van Hemel SB (eds) (2008). Behavioral Modeling and Simulation [Electronic Resource]: From Individuals to Societies. National Academies Press: Washington DC.

Received 19 November 2012; accepted 14 August 2014 after three revisions 